\title{
Clinical applications of nucleic acid aptamers in cancer (Review)
}

\author{
XIAOYU PEI ${ }^{1}$, JUN ZHANG $^{1}$ and JIE LIU ${ }^{1,2}$ \\ ${ }^{1}$ Department of Digestive Diseases, Huashan Hospital, Fudan University, Shanghai 200040; \\ ${ }^{2}$ Institute of Biomedical Sciences and Department of Immunology of Shanghai Medical School, \\ Fudan University, Shanghai 200032, P.R. China
}

Received October 24, 2013; Accepted January 28, 2014

DOI: $10.3892 / \mathrm{mco} .2014 .255$

\begin{abstract}
Nucleic acid aptamers are small single-stranded DNA or RNA oligonucleotide segments, which bind to their targets with high affinity and specificity via unique three-dimensional structures. Aptamers are generated by an iterative in vitro selection process, termed as systematic evolution of ligands by exponential enrichment. Owing to their specificity, non-immunogenicity, non-toxicity, easily modified chemical structure and wide range of targets, aptamers appear to be ideal candidates for various clinical applications (diagnosis or treatment), such as cell detection, target diagnosis, molecular imaging and drug delivery. Several aptamers have entered the clinical pipeline for applications in diseases such as macular degeneration, coronary artery bypass graft surgery and various types of cancer. The aim of this review was to summarize and highlight the clinical applications of aptamers in cancer diagnosis and treatment.
\end{abstract}

\section{Contents}

1. Introduction

2. Aptamers and SELEX

3. Clinical applications of aptamers in cancer

4. Conclusion and future perspectives

\section{Introduction}

Cancer is one of the leading causes of mortality worldwide. A statistical survey conducted in 2008 reported $\sim 12.7$ million cancer cases and 7.6 million cancer-related deaths worldwide (1). An estimated total of 1,529,560 new cancer cases and 569,490 cancer-related deaths occurred in 2010 (2). Over

Correspondence to: Dr Jie Liu, Department of Digestive Diseases, Huashan Hospital, Fudan University, 12 Middle Wulumuqi Road, Shanghai 200040, P.R. China

E-mail: jieliu@fudan.edu.cn

Key words: aptamer, clinical application, cancer diagnosis, cancer therapy, systematic evolution of ligands by exponential enrichment the last several decades, significant investments and efforts have focused on battling cancer, making it a top priority in the pharmaceutical industry and the global Institutes of Health. With the development of novel anticancer agents with greater efficacy and fewer side effects, accurate and efficient delivery of these agents to the tumor sites is of utmost importance (3). In order to design an ideal and effective anticancer drug, several aspects have to be optimized simultaneously, including toxicity, side effects, targeting, delivery and controlled release. Although a large number of anticancer drugs have been approved by the Food and Drug Administration (FDA) (4), the majority are not molecularly targeted, which means that developing novel drugs with higher efficacy but less toxicity and fewer side effects is a major aim for cancer therapy.

Aptamers are small single-stranded (ss) DNA or RNA oligonucleotides (with a molecular weight of 5-40 kDa), which fold into well-defined three-dimensional (3D) structures due to various intramolecular interactions (5). Aptamers bind onto their targets with high affinity and specificity and are traditionally generated through systematic evolution of ligands by exponential enrichment (SELEX) (6,7). Aptamers have been selected against a wide range of targets, similar to antibodies, which may be crafted to bind onto multiple and different targets $(5,8)$, such as proteins, phospholipids, sugars, nucleic acids and whole cells. However, in comparison to antibodies, aptamers possess the following advantages: i) they are of small size and lower complexity, with low immunogenicity; ii) they are easier to synthesize and modify in vitro; iii) they exhibit higher affinity and specificity for their targets; iv) their structural flexibility enables aptamers to bind onto hidden epitopes, which cannot be targeted by antibodies (9); and v) they exhibit higher stability, with the potential to be stored easily until use (Table I). Over the last two decades, since aptamers were first selected through SELEX, they have quickly emerged as a novel and powerful class of ligands with an excellent potential for diagnostic and therapeutic applications (10). As the use of aptamers has been extended from the basic biology of cell processes and gene regulation to therapeutic and diagnostic applications, numerous patented aptamers are currently being tested in clinical trials and were recently reviewed (9). Pegaptanib (Pfizer, New York, NY, USA / EyeTech, Melville, NY, USA), an aptamer that binds to human vascular endothelial growth factor (VEGF), has been approved by the FDA for clinical use in treating age-related macular degeneration (AMD) and has already been proven to be a milestone for the 
Table I. Advantages of nucleic acid aptamers.

\begin{tabular}{ll}
\hline No. & \multicolumn{1}{c}{ Advantages } \\
\hline 1 & High binding affinity and specificity \\
2 & Ease of synthesis and modification \\
3 & Low immunogenicity and no toxicity \\
4 & Small size and stable structure \\
5 & Structural flexibility and ease of storage
\end{tabular}

applications of aptamer technology. In this review, we focused on recent developments in aptamer technology, as well as their applications in cancer diagnosis and therapy.

\section{Aptamers and SELEX}

Overview of aptamers. By definition, aptamers are synthetic, highly structured, small, typically $<100$ mer, ssDNA or RNA ligands. The term 'aptamer' means 'to fit' (aptus) in Latin (6), which indicates two important properties of aptamers: i) their ability to fold into complex tertiary structures and recognize their targets with high affinity (low nM to high $\mathrm{pM}$ equilibrium dissociation constants); and ii) their specificity, somewhat analogous to antigen-antibody interactions. Using this technique, a number of aptamers that specifically recognize targets, such as metal ions (11-13), organic dyes and amino acids (14-17), antibiotics $(18,19)$ and peptides $(20,21)$, as well as proteins of various sizes and functions $(6,22,23)$, whole cells (24-27), whole organisms (28), viruses (29) and bacteria (30), have been obtained (31-33). The SELEX process is based on the ability of these small oligonucleotides to fold into unique 3D structures that interact with a target with high specificity and affinity through such interactions as van der Waals surface contacts, hydrogen bonding and base stacking. Starting with a library containing random RNA sequences with $\sim 10$ (34-36) varieties of RNA molecules, in vitro binding, elution and reverse polymerase chain reaction (PCR) amplification techniques allow for the selection of RNA molecules that efficiently bind to a specific receptor or ligand with high affinity $(37,38)$. Conceptually, the marked specificity and high affinity of aptamers to a wide variety of targets, coupled with the ease of design and molecular engineering, as mentioned above, have made them broadly popular and highly suitable for development as clinical diagnostic and therapeutic agents in cancer research (39-41). Pegaptanib (Pfizer/Eyetech), an aptamer targeting VEGF, has already been approved by the FDA for clinical use in treating AMD (42). A variety of aptamers against other molecular targets are currently under clinical investigation (10), with several more in the pipeline.

In vitro SELEX. Aptamers are identified through an in vitro process, which specifically isolates aptamers for a target of interest involving iterative rounds, termed as SELEX. Briefly, for the SELEX process, as shown in Fig. 1, a random aptamer or oligonucleotide library pool (DNA or RNA) is incubated with the target of interest, with heating and cooling to promote formation of stable structures. After washing, the bound sequences are eluted and incubated with a control target (if negative selection is required) to remove sequences that exhibit recognition with the control as well. The protein-bound aptamers are then recovered. These sequences are amplified with PCR or reverse transcription-PCR. ssRNA or DNA sequences representing the recovered sequences are then generated from these PCR products and used in the subsequent selection round. The process is repeated until the pool is enriched for sequences that specifically recognize the target. The enriched pool is cloned and then sequenced to obtain the individual sequences known as aptamers (7). As this is an in vitro process, the selection conditions may be manipulated to obtain aptamers with properties desirable for a particular purpose, i.e., aptamers that bind to a target at different temperatures and buffer compositions may be used for different purposes, or modified oligonucleotide bases which may be introduced to enhance stability.

Cell-SELEX. The cancer cell surface represents a complex environment with a vast array of potential targets of diagnostic and therapeutic interest; therefore, the aptamers selected by SELEX may exhibit low or no affinity towards these targets, due to shielding of the aptamer binding domain. To overcome this hindrance, a process called Cell-SELEX (cell-based selection of aptamers specific to cancer cells) has been reported to isolate aptamers from living cells as targets, such as cancer cells (24). In Cell-SELEX, instead of using purified protein targets, whole living cells are used as the targets. To generate aptamers that specifically target cancer cells, a library of ssDNA is used (24). This library, which has a random sequence of 30-40 bases and a region flanked by primer sequences of 18-20 bases, is incubated with the target cells. The library and forward primers are labeled with a fluorophore, so that the sense strand of the PCR product for each round, which serves as the the library for the next round, is also fluorescently labeled, enabling the entire process to be monitored by flow cytometry. After washing, the DNA sequences bound to the target cell surface are collected and then incubated with the negative control cells. All the DNA sequences that bind to the negative control cells are removed. To avoid recognition of normal cells, the aptamers bound to these non-specific proteins are removed. The remaining sequences are amplified for the next round of selection. Generally, 20 rounds of Cell-SELEX are required to isolate aptamers with the highest selective affinity to the target cells.

A panel of aptamer probes has been successfully selected by Cell-SELEX (Fig. 1) for several types of cancer cells, including lymphocytic leukemia, myeloid leukemia, liver cancer, small- and non-small-cell lung cancer cells (25-27). All these aptamers were found to exhibit high affinity and specific selectivity for their targets. Those results illustrated how aptamers may be selected without specific knowledge of the molecular signature of the cell or the number or type of proteins on the cell surface. Moreover, it is possible to profile the molecular characteristics of the target cancer type, which has been a main objective in the development of cell-based aptamers for use in cancer diagnosis and treatment. The applications of aptamers for cancer diagnosis and therapy through cancer cell detection, cancer cell imaging and targeted drug delivery are discussed below. 
Table II. Clinical application of aptamers in cancer.

\begin{tabular}{llllr}
\hline Aptamers & Target & Nucleic acid & \multicolumn{1}{c}{ Clinical applications } \\
\hline A9, A10 & PSMA & RNA & Prostate cancer imaging and therapy \\
TTA1 & Tenascin C & RNA & Cancer imaging & $(67-74)$ \\
AS1411 & Nucleolin & DNA & Cancer imaging and therapy & $(79,80)$ \\
AptA, AptB & Mucin 1 & DNA & Cancer imaging and therapy \\
NOX-A12 & CXCL12 & RNA & Hematological cancer therapy & $(84-86)$
\end{tabular}

PSMA, antiprostate-specific membrane antigen; CXCL12, chemokine (C-X-C motif) ligand 12.

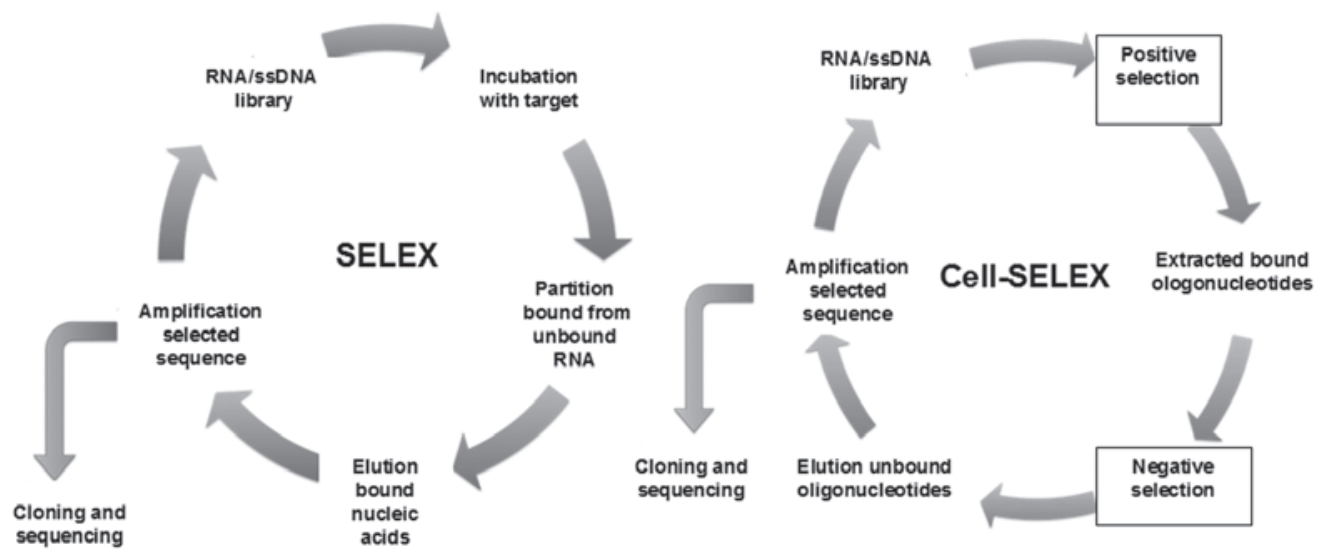

Figure 1. A schematic depiction of systematic evolution of ligands by exponential enrichment (SELEX) and Cell-SELEX (cell-based selection of aptamers specific to cancer cells). The major differences between SELEX and Cell-SELEX are marked by rectangles.

\section{Clinical applications of aptamers in cancer}

Cancer is a class of diseases that originate from mutations and alterations at the genetic and, subsequently, the molecular level. However, at the precancerous and early stages of cancer, detection is limited due to the low number of tumor cells and molecular markers. Furthermore, to a certain degree, detection may exert a negative effect on cancer therapy. Therefore, determining the molecular characteristics of cancer, particularly the characteristic proteins associated with a specific type of cancer, may prove beneficial for clinical diagnosis and therapy (43), particularly during the early stage of tumorigenesis and cancer progression. By contrast, the traditional diagnostic methods, such as computed tomography (CT), magnetic resonance imaging (MRI) and positron emission tomography with radiolabeled 2-fluoro-deoxyglucose, only assess anatomical changes or non-specific glucose metabolism (44).

Aptamers selected by SELEX have shown the high sensitivity and specificity required of bioprobes used for the accurate and early diagnosis of tumors. With their high-recognition specificity, aptamers can accurately distinguish between different types and even subtypes of cancer cells (45). Moreover, aptamers targeting wide-range receptors have been extensively adapted for disease diagnosis and the delivery of therapeutic moieties into cells. In 2000, Hicke et al (46) coined the term 'escort aptamers' and demonstrated that aptamers may be used as tools to deliver therapeutic and diagnostic reagents. A wide range of molecular species have been transported intracellularly using these nanoscale delivery vehicles that include small interfering RNAs (siRNAs), drugs, toxins, enzymes, photodynamic molecules and radionuclides. Intracellular delivery of these compounds may be achieved by employing aptamers for internalized cell surface receptors. Furthermore, to achieve delivery selectivity to the diseased cells, the cell surface receptor selected is either a tumor biomarker or an aberrantly expressed surface protein unique to the target cell. Aptamer-mediated targeted detection and delivery increases the positive prognosis and sensibility of early diagnosis, correspondingly decreasing treatment costs due to the reduced incidence of late-stage cancers and increased number of therapeutic options, while reducing the side effects. The examples of aptamer-mediated clinical applications in cancer, which are discussed below, are summarized in Table II.

Aptamer-based cancer cell detection and imaging. The binding affinity of aptamers to their targets enables the detection of cancer cells at low levels, resulting in an early and sensitive diagnosis. In addition, DNA molecules have the advantage of predictable structure and easy site-specific chemical modification. Therefore, aptamers may be quickly and reproducibly synthesized with fluorescent molecules and nanoparticles (NPs) conjugated for targeted cells and tissue detection and imaging.

It is well known that gold nanomaterials possess unusual optical and electronic properties, high stability and biological 
compatibility, controllable morphology and size dispersion, and easy surface functionalization (47-51) from the standpoints of engineering and application. Aptamer-conjugated gold NPs (Apt-AuNPs) provide a powerful platform to facilitate targeted recognition and detection. By using Apt-AuNPs, a colorimetric assay for the direct detection of cancer cells has been developed (52). Aptamers specific to their targets on the cell membrane surface were conjugated to gold NPs. These Apt-AuNPs were then targeted to assemble on the cell surface membrane through the recognition of the aptamers to their targets. The assembly of the Apt-AuNPs around the cell surface causes a shift in the extinction spectra of the particles when the AuNPs are in sufficient proximity for their surface plasmon resonances to overlap. This assay exhibits a significantly high sensitivity, with a detection limit for simple absorbance of just 90 cells. When the number of target cells increases to 1,000 , the color change reaction may be observed by the naked eye. This method demonstrates the potential of aptamer-based analysis for rapid, simple, direct and sensitive detection of cancer cells in clinical diagnosis. In addition, using the two-photon scattering (TPS) technique, Lu et al (53) captured the signal from Apt-AuNP-based cell detection, resulting in the highly selective and sensitive detection of the SK-BR-3 breast cancer cell line at a level of 100 cells $/ \mathrm{ml}$, using multifunctional (monoclonal anti-HER 2/c-erb-2 antibody and S6 RNA aptamer) AuNP conjugates. Oval-shaped AuNPs were introduced instead of spherical AuNPs. As the particle aspect ratio increased, the TPS intensity change became higher, resulting in improved sensitivity (53).

The research on the applications of aptamer-conjugated fluorophores has advanced rapidly over the last few years (54), including the use of quantum-dot (QD)-conjugated aptamers to perform multiplex detection of cancer cells (44). In that study, three aptamers (TTA1, AS1411 and Muc1) were conjugated to QDs with emission wavelengths of 605,655 and $705 \mathrm{~nm}$, respectively; for the confocal microscopic analysis of multiplex imaging of cancer, healthy and disease cell lines were incubated with each QD-conjugate. As expected, fluorophore-labeled aptamers were able to detect and differentiate between different types of cancer cells and produced a visible fluorescence signal in the presence of target cells, which indicated that aptamers have high potential as a clinical diagnostic tool.

Gold nanorods (NRs) have been utilized as a platform for multiple aptamer immobilization to detect cancer cells (55). Through covalent linkages of fluorophore-labeled aptamers on the NR surface, $\leq 80$ fluorophore-labeled aptamers may be attached on a single NR, causing a 26 -fold higher affinity and $>300$-fold higher fluorescence signal. In this case, the use of NRs as a nanoplatform for multivalent binding of aptamers increases the signal as well as and binding strength of these aptamers in cancer cell recognition. As a consequence of the selective recognition properties of aptamers, this technique may be used in clinical detection to enhance binding affinity and signaling strength when the concentration of target cells is relatively low.

With increasing detection sensitivity, a dye-doped silica NPs-based method for sensitive and rapid detection of cancer cells was constructed (56). To achieve specific recognition, the biotinylated aptamers are immobilized onto the surfaces of neutravidin-coated fluorescent NPs (FNPs) to form aptamer-conjugated FNPs (Apt-FNPs), which have demonstrated low background signal, high signal enhancement and efficient functionalization. The FNPs are then functionalized with polyethylene glycol (PEG) to prevent non-specific interactions with neutravidin and allow universal binding with biotinylated molecules. Multiplexed cancer cells may also be detected by employing a combination of fluorescence resonance energy transfer of FNPs and the conjugation of different aptamers (57). Furthermore, single-, dual- and triple-dye-doped silica NPs were prepared according to this technology (58). This method provided an additional potential candidate for cancer cell imaging.

In addition to cancer cell detection, improved molecular imaging techniques together with novel near-infrared fluorescence probes, which may also be attached to aptamers, allow in vivo multiparameter $3 \mathrm{D}$ exploration of tumors, even deep inside the tissue (59). Aptamer-mediated technology is likely to gain applications in clinical cancer diagnosis, with cancer cell detection as well as cancer cell and tissue visualization. Fluorescein isothiocyanate (FITC)-labeled aptamers were used for tissue staining and imaging under confocal microscopy (60). Compared to the negative control (FITC-labeled unselected library), targeted tissue was recognized with fluorescence signal following incubation with FITC-labeled aptamers, which indicated aptamers as potential molecular probes for cancer visualized diagnosis. Other methods were also developed for labeling aptamers for flow cytometry applications $(23,61)$. Flow cytometric analysis was employed to compare binding of a FITC-labeled DNA aptamer with a complex of mouse antihuman neutrophil elastase (HNE) antibody and FITC-labeled rat antimouse antibody to HNE-labeled beads. The results revealed that aptamers and antibodies were equally efficient in HNE detection (61). Further studies demonstrated that, based on aptamers' high specificity of target recognition, fluorescein-labeled aptamers were selective in flow cytometric experiments for binding to human recombinant CD4-stained mouse $\mathrm{T}$ cells expressing human $\mathrm{CD} 4$, but did not detect control mouse $\mathrm{T}$ cells lacking human CD4 (23).

For cancer cell imaging, the antiprostate-specific membrane antigen (PSMA) aptamer was used. Due to its high affinity and specificity, this aptamer is able to mediate cell-specific endocytosis of the NPs upon molecular recognition between the aptamer and its receptors. The synthesized aptamer-functionalized QDs and super-paramagnetic iron oxide NPs were used for the recognition of cell receptors as well as optical imaging or MRI $(62,63)$. The same concept was also applied to functionalize gold NPs with anti-PSMA aptamer for CT. The results demonstrated that the functionalized gold NPs may enhance the CT image by 4 -fold (64). For further study in vivo, molecular imaging of tumors inside mice was performed. TD05, an aptamer that specifically binds Ramos cells, was modified with fluorophores, successfully exhibiting aptamer fluorescence overlapping the tumor site in a mouse cancer model under a whole-animal imaging system.

Overall, the studies discussed in this section helped expedite the process of using aptamers in the clinical setting for cancer diagnosis. 
Aptamer-mediated therapy for cancer. Several aptamers, including pegaptanib and REG1, have already been used for clinical treatment (42).

From a therapeutic point of view, a useful therapeutic agent may stop being used when a more cost-effective, safer or simply generic product is released. Aptamers, targeting a broad range of receptors, with numerous ideal properties, such as high specificity and affinity, easy modification, easy synthesis, stability, low immunogenicity and toxicity, have emerged as promising therapeutic reagents.

PSMA, a transmembrane protein that is highly expressed in human prostate cancer cells and the vascular endothelium, is an attractive target, as it is abundantly expressed on the surface of prostate cancer cells, is known to internalize certain ligands, possesses enzymatic properties as a glutamate carboxypeptidase and is a verified target of a clinically applied imaging agent for prostate cancer (65). In 2002, a 2'-fluorpyrimidine RNA aptamer screen was performed and two stabilized RNA aptamers, A9 and A10, which were capable of binding PSMA with low nanomolar affinity, were first identified (66). These aptamers have been applied to several anticancer preclinical targeting studies of NPs, toxins, therapeutics and siRNAs. siRNAs are double-stranded oligonucleotides (21-23 bp) that bind specifically to cognate mRNA and promote its degradation, effectively decreasing the expression of the target gene product. To develop its ability, a siRNA has to be efficiently delivered to the intracellular space in vivo. For this purpose, an aptamer-siRNA chimera construct was generated to combine the RNA aptamer A10, specific for PSMA, with a siRNA against the survival factors polo-like kinase 1 (Plk-1) and B-cell lymphoma 2 (Bcl-2), which are two antiapoptotic genes involved in cell survival and are commonly overexpressed in human tumors. After being injected at the site of a tumor, aptamer-Plk-1 or Bcl-2 siRNA chimeras were shown to deliver the siRNAs effectively to PSMA-expressing cells, inducing apoptosis in targeted cancer cells and tumor regression in animal models of prostate cancer (67).

An improved treatment of prostate cancer was aptamer-short hairpin RNA (shRNA) chimera, a PSMA-targeted aptamer-conjugated shRNA, which was used to attenuate DNA-repair pathways, resulting in tissue-selective sensitization to ionizing radiation (IR) therapy (68). A10-3-shRNAs targeting the catalytic subunit of the DNA protein kinase (DNAPK) caused a selective reduction of DNAPK RNA and protein in PSMA-positive cells, xenografts and human prostatic tissues. Intratumorally, the administration of aptamer-targeted DNAPK shRNAs combined with IR significantly and specifically enhanced PSMA-positive tumor response to IR, leading to decreased DNA-repair and cancer cell survival following chemotherapy or radiation treatment.

The application of antitumor agents, such as docetaxel, dextran, cisplatin, daunorubicin and doxorubicin, is limited due to their side-effects and toxicity. The first report of targeted drug delivery with NP-aptamer bioconjugates was described by Farokhzad et al (69). The anti-PSMA A10 aptamer, or its truncated version A10-3, was used to target NPs, demonstrating that the A10-3 aptamer may be used to target poly(lactic acid)-block PEG copolymer NPs to PSMA-positive prostate cancer cells (69). Furthermore, the A10-3 aptamer was modified by poly(D,L-lactic-co-glycolic acid; PLGA) NPs to deliver docetaxel (Dtxl); an antimitotic chemotherapy drug (70) to prostate tumors in vivo. When compared with non-targeted NPs that lacked the anti-PSMA aptamer, the Dtxl-NP-Apt conjugates mediated targeted uptake and controlled release of drugs, resulting in potent efficacy and reduced toxicity after intratumoral injection (71). In an extensive study, following systemic administration, A10-3 aptamer induced tumor accumulation of PLGA NPs by 3.7-fold when compared to the non-targeted particle (72). Further investigations demonstrated that the NP-aptamer bioconjugates may be precisely engineered and formulated by controlling size, composition, polydispersity, aptamer density and drug loading, thereby resulting in the desired functions and biodistribution required for further clinical development (72-74). Another polymeric formulation has been used to deliver cisplatin to PSMA expressing tumors, whereas a dosage of $0.3 \mathrm{mg} / \mathrm{kg}$ of aptamer-targeted cisplatin NPs was more efficacious compared to a $1 \mathrm{mg} / \mathrm{kg}$ of free cisplatin (75). These preclinical studies demonstrated the potential of aptamers as therapeutic reagents to efficiently deliver drugs to targeted cancers. In addition, these results also indicated aptamers as targeting ligands to alter the biodistribution, tumor uptake and tumor retention of therapeutic NP reagents.

The AS1411 aptamer against nucleolin, a Bcl-2 mRNA-binding protein expressed on the surface of several cancer cell types, including those associated with acute myeloid leukemia (AML), has demonstrated promising antineoplastic effects, which may be attributed to interference with the multitude of nucleolin-mediated cell processes. AS1411, a DNA aptamer, was shown to interfere with DNA replication, causing S-phase arrest (76), as well as with the stabilization of Bcl-2 mRNA, a known inhibitor of apoptosis, suggesting another possible mechanism of action (77). The use of AS1411-conjugated NPs for drug delivery was performed on MCF-7 breast cancer cells which overexpress nucleolin, exhibiting increased cell death compared to that of cancer cells that do not overexpress nucleolin. Moreover, the extent of delivery and dosage may be controlled using complementary DNA of the aptamer as an antidote (78). Furthermore, in vitro and in vivo, AS1411 has demonstrated growth inhibitory effects and antitumor activity against multiple cancer cell lines and tumor tissues, respectively. The subsequent toxicity studies in rats and dogs exhibited a favorable safety profile, prompting the initiation of human clinical trials. Notably, internalization of the aptamer results in a decrease in the expression of several cancer-associated mRNAs and, therefore, reduced cell proliferation.

Completed phase I trials demonstrated that AS1411 exhibited minimal toxicity in 17 patients with advanced solid tumors. Moreover, there was evidence of promising clinical activity, with 7 patients achieving disease stabilization and 1 patient with renal cell cancer achieving a complete response by 11 months (79). Phase II studies on metastatic renal cell cancer and AML are currently underway. The development and clinical trial data were reviewed by Mongelard and Bouvet (80).

Mucin 1 (Muc1) is a glycoprotein found on the surface of normal epithelial cells and functions as a barrier against microorganisms and degradative enzymes (81). Muc1 is released into the bloodstream and has been successfully used as a tumor marker for bladder cancer. In addition, Mucl is an attractive target, as its overexpression has been associated with various types of cancer and its distribution pattern changes on 
the cell surface $(82,83)$. DNA aptamers directed against Muc1 (AptA and AptB) were identified in 2006 (84) and have since been implemented in ex vivo imaging and radiotherapy (85). In subsequent studies, when such an DNA aptamer was coupled to the light-activated photodynamic therapy agent chlorine e6, it specifically delivered chlorine e6 into epithelial cancer cells, with a significant enhancement in efficiency $(>500$-fold increase) compared to that of the drug alone, and achieved cancer-specific cytotoxicity (86). These results suggested the anti-Muc1 aptamers may function as drug carriers to specific epithelial cancer cells. As target QD scaffolds allow for labelling of a single QD with multiple aptamers, the efficiency of binding anti-Muc1 aptamers with QDs may be enhanced by avidity (87). In a mouse model bearing A2780/AD human ovarian cancer xenografts, an increased amount of QD-Muc1 accumulated in the tumors compared to non-modified QD. This data support that anti-Mucl aptamer targeting may be a therapeutic candidate for drug delivery when improved by the avidity associated with NP decoration.

NOX-A12 is a 45-nt L-RNA aptamer, based on L-ribose, which is clinically developed by NOXXON Pharma AG (Berlin, Germany) (88). NOX-A12 was selected against stromal cell-derived factor-1 (SDF-1), which binds to chemokine (C-X-C motif) ligand 12, a chemokine that is involved in tumor metastasis, angiogenesis, cell homing and tissue regeneration (89). Inhibition of the SDF-1 by NOX-A12 may prove useful in the treatment of several types of cancer. NOX-A12 is currently in phase I clinical trials for the treatment of lymphoma, multiple myeloma and hematopoietic stem cell transplantation.

The ultimate aim in cancer therapy remains the development of treatment modalities that effectively eliminate cancer cells without affecting normal cells. Due to their uniqueness and high specificity, aptamers and aptamer-based drug delivery systems are promising tools for targeted cancer therapy. Several more aptamers may soon be joining pegaptanib in the field of cancer diagnosis and treatment.

\section{Conclusion and future perspective}

Aptamers have become increasingly important molecular tools for diagnosis and therapeutics and aptamer-based technology holds promise for the advancement of targeted cancer diagnosis and therapy. Notably, due to their unique pharmacokinetic properties, aptamers may be exploited for various purposes. One highly attractive characteristic of aptamers is that they are amenable to chemical modification and bioconjugation to various moieties, such as NPs, imaging agents, siRNAs and therapeutic drugs. As discussed in this review, aptamers were clinically proven as inhibitors with great promise as targeting ligands for treating or diagnosing cancer. Further maturation of the field of nanotechnology and RNA interference may produce more sophisticated, multifunctional constructs, capable of improved selective cytotoxicity. Moreover, recent studies demonstrated improved binding characteristics for multivalent aptamer constructs over their monovalent counterparts; therefore, it is likely that this will become a more popular approach for delivery applications. The continued development of animal models for the evaluation of safety and efficacy of these constructs is expected to lay the foundation for their increased use in humans. Encouraging results from aptamer clinical trials and successful outcomes, including the development of pegaptanib sodium (Macugen), have further strengthened the optimism surrounding their development and use as therapeutic and diagnostic reagents. Although aptamers are relatively new to the clinical setting, their versatility and numerous aptamer-based therapeutic approaches discussed above suggest that this technology may have a substantial impact on patient care in the following years. Overall, aptamers offer a promising and unique technology that may be applied in clinical diagnostics and therapeutics. Their rapid development over the last two decades is notable, indicating that aptamers may become a valuable tool for clinicians in the near future.

\section{Acknowledgements}

This study was supported by a grant from the Ministry of Science and Technology of China (no. 2010CB732405).

\section{References}

1. Jemal A, Bray F, Center MM, et al: Global cancer statistics. CA Cancer J Clin 61: 69-90, 2011.

2. Jemal A, Siegel R, Xu J and Ward E: Cancer statistics, 2010. CA Cancer J Clin 60: 277-300, 2010.

3. Rajendran L, Knolker HJ and Simons K: Subcellular targeting strategies for drug design and delivery. Nat Rev Drug Discov 9: 29-42, 2010.

4. Blagosklonny MV: Analysis of FDA approved anticancer drugs reveals the future of cancer therapy. Cell Cycle 3: 1035-1042, 2004.

5. Kanwar JR, Mohan RR, Kanwar RK, et al: Applications of aptamers in nanodelivery systems in cancer, eye and inflammatory diseases. Nanomedicine (Lond) 5: 1435-1445, 2010.

6. Ellington AD and Szostak JW: In vitro selection of RNA molecules that bind specific ligands. Nature 346: 818-822, 1990.

7. Tuerk C and Gold L: Systematic evolution of ligands by exponential enrichment: RNA ligands to bacteriophage T4 DNA polymerase. Science 249: 505-510, 1990.

8. Keefe AD and Cload ST: SELEX with modified nucleotides. Curr Opin Chem Biol 12: 448-456, 2008.

9. Majumder P, Gomes KN and Ulrich H: Aptamers: from bench side research towards patented molecules with therapeutic applications. Expert Opin Ther Pat 19: 1603-1613, 2009.

10. Keefe AD, Pai S and Ellington A: Aptamers as therapeutics. Nat Rev Drug Discov 9: 537-550, 2010.

11. Ciesiolka J and Yarus M: Small RNA-divalent domains. RNA 2: 785-793, 1996.

12. Hofmann HP, Limmer S, Hornung V and Sprinzl M: $\mathrm{Ni}^{2+}$-binding RNA motifs with an asymmetric purine-rich internal loop and a G-A base pair. RNA 3: 1289-1300, 1997.

13. Rajendran M and Ellington AD: Selection of fluorescent aptamer beacons that light up in the presence of zinc. Anal Bioanal Chem 390: 1067-1075, 2008.

14. Geiger A, Burgstaller P, von der Eltz H, et al: RNA aptamers that bind L-arginine with sub-micromolar dissociation constants and high enantioselectivity. Nucleic Acids Res 24: 1029-1036, 1996.

15. Connell GJ, Illangesekare M and Yarus M: Three small ribooligonucleotides with specific arginine sites. Biochemistry 32: 5497-5502, 1993.

16. Mannironi C, Scerch C, Fruscoloni P and Tocchini-Valentini GP: Molecular recognition of amino acids by RNA aptamers: the evolution into an L-tyrosine binder of a dopamine-binding RNA motif. RNA 6: 520-527, 2000.

17. Harada K and Frankel AD: Identification of two novel arginine binding DNAs. EMBO J 14: 5798-5811, 1995.

18. Wallis MG, Streicher B, Wank $\mathrm{H}$, et al: In vitro selection of a viomycin-binding RNA pseudoknot. Chem Biol 4: 357-366, 1997.

19. Wallace ST and Schroeder R: In vitro selection and characterization of streptomycin-binding RNAs: recognition discrimination between antibiotics. RNA 4: 112-123, 1998.

20. Nieuwlandt D, Wecker M and Gold L: In vitro selection of RNA ligands to substance P. Biochemistry 34: 5651-5659, 1995. 
21. Williams KP, Liu XH, Schumacher TN, et al: Bioactive and nuclease-resistant L-DNA ligand of vasopressin. Proc Natl Acad Sci USA 94: 11285-11290, 1997.

22. Mallikaratchy P, Stahelin RV, Cao Z, et al: Selection of DNA ligands for protein kinase C-delta. Chem Commun (Camb) 30 3229-3231, 2006.

23. Davis KA,Lin Y,Abrams B and Jayasena SD: Staining of cell surface human CD4 with 2'-F-pyrimidine-containing RNA aptamers for flow cytometry. Nucleic Acids Res 26: 3915-3924, 1998.

24. Shangguan D, Li Y, Tang Z, et al: Aptamers evolved from live cells as effective molecular probes for cancer study. Proc Natl Acad Sci USA 103: 11838-11843, 2006.

25. Tang Z, Shangguan D, Wang K, et al: Selection of aptamers for molecular recognition and characterization of cancer cells. Anal Chem 79: 4900-4907, 2007.

26. Chen HW, Medley CD, Sefah K, et al: Molecular recognition of small-cell lung cancer cells using aptamers. ChemMedChem 3 991-1001, 2008

27. Sefah K, Tang ZW, Shangguan DH, et al: Molecular recognition of acute myeloid leukemia using aptamers. Leukemia 23 : 235-244, 2009

28. Lorger M, Engstler M, Homann M and Goringer HU: Targeting the variable surface of African trypanosomes with variant surface glycoprotein-specific, serum-stable RNA aptamers Eukaryot Cell 2: 84-94, 2003.

29. Tang Z, Parekh P, Turner P, et al: Generating aptamers for recognition of virus-infected cells. Clin Chem 55: 813-822, 2009.

30. Bruno JG and Kiel JL: In vitro selection of DNA aptamers to anthrax spores with electrochemiluminescence detection. Biosens Bioelectron 14: 457-464, 1999.

31. Shu D and Guo P: A viral RNA that binds ATP and contains a motif similar to an ATP-binding aptamer from SELEX. J Biol Chem 278: 7119-7125, 2003.

32. Clark SL and Remcho VT: Aptamers as analytical reagents Electrophoresis 23: 1335-1340, 2002.

33. Bouvet P: Determination of nucleic acid recognition sequences by SELEX. Methods Mol Biol 148: 603-610, 2001

34. Sablin EP and Fletterick RJ: Nucleotide switches in molecular motors: structural analysis of kinesins and myosins. Curr Opin Struct Biol 11: 716-724, 2001

35. Shenton W, Pum D, Sleytr UB and Mann S: Synthesis of cadmium sulphide superlattices using self-assembled bacterial S-layers. Nature 389: 585-587, 1997.

36. Yeakley JM, Fan JB, Doucet D, et al: Profiling alternative splicing on fiber-optic arrays. Nat Biotechnol 20: 353-358, 2002

37. Klug SJ and Famulok M: All you wanted to know about SELEX Mol Biol Rep 20: 97-107, 1994.

38. Ciesiolka J, Gorski J and Yarus M: Selection of an RNA domain that binds $\mathrm{Zn}^{2+}$. RNA 1: 538-550, 1995.

39. Nimjee SM, Rusconi CP, Harrington RA and Sullenger BA: The potential of aptamers as anticoagulants. Trends Cardiovasc Med 15: 41-45, 2005.

40. Levy-Nissenbaum E, Radovic-Moreno AF, Wang AZ, et al: Nanotechnology and aptamers: applications in drug delivery. Trends Biotechnol 26: 442-449, 2008

41. Thiel KW and Giangrande PH: Therapeutic applications of DNA and RNA aptamers. Oligonucleotides 19: 209-222, 2009.

42. Dyke CK, Steinhubl SR, Kleiman NS, et al: First-in-human experience of an antidote-controlled anticoagulant using RNA aptamer technology: a phase 1a pharmacodynamic evaluation of a drug-antidote pair for the controlled regulation of factor IXa activity. Circulation 114: 2490-2497, 2006.

43. Smith JE, Medley CD, Tang Z, et al: Aptamer-conjugated nanoparticles for the collection and detection of multiple cancer cells. Anal Chem 79: 3075-3082, 2007.

44. Kang WJ, Chae JR, Cho YL, et al: Multiplex imaging of single tumor cells using quantum-dot-conjugated aptamers. Small 5: 2519-2522, 2009.

45. Zhao Z, Xu L, Shi X, et al: Recognition of subtype non-small cell lung cancer by DNA aptamers selected from living cells. Analyst 134: 1808-1814, 2009.

46. Hicke BJ and Stephens AW: Escort aptamers: a delivery service for diagnosis and therapy. J Clin Invest 106: 923-928, 2000.

47. Daniel MC and Astruc D: Gold nanoparticles: assembly, supramolecular chemistry, quantum-size-related properties, and applications toward biology, catalysis, and nanotechnology. Chem Rev 104: 293-346, 2004.

48. Rosi NL, Giljohann DA, Thaxton CS, Lytton-Jean AK, et al: Oligonucleotide-modified gold nanoparticles for intracellular gene regulation. Science 312: 1027-1030, 2006.
49. Sperling RA, Rivera Gil P, Zhang F, et al: Biological applications of gold nanoparticles. Chem Soc Rev 37: 1896-1908, 2008.

50. Storhoff JJ,Lucas AD, Garimella V, et al: Homogeneous detection of unamplified genomic DNA sequences based on colorimetric scatter of gold nanoparticle probes. Nat Biotechnol 22: 883-887, 2004.

51. Grzelczak M, Perez-Juste J, Mulvaney P and Liz-Marzan LM: Shape control in gold nanoparticle synthesis. Chem Soc Rev 37: 1783-1791, 2008

52. Medley CD, Smith JE, Tang Z, et al: Gold nanoparticle-based colorimetric assay for the direct detection of cancerous cells. Anal Chem 80: 1067-1072, 2008.

53. Lu W, Arumugam SR, Senapati D, et al: Multifunctional oval-shaped gold-nanoparticle-based selective detection of breast cancer cells using simple colorimetric and highly sensitive two-photon scattering assay. ACS Nano 4: 1739-1749, 2010.

54. Zhang J, Jia X, Lv XJ, et al: Fluorescent quantum dot-labeled aptamer bioprobes specifically targeting mouse liver cancer cells. Talanta 81: 505-509, 2010.

55. Huang YF, Chang HT and Tan W: Cancer cell targeting using multiple aptamers conjugated on nanorods. Anal Chem 80 $567-572,2008$

56. Estevez MC, O'Donoghue MB, Chen X and Tan W: Highly fluorescent dye-doped silica nanoparticles increase flow cytometry sensitivity for cancer cell monitoring. Nano Res 2: 448-461, 2009.

57. Chen X, Estevez MC, Zhu Z, et al: Using aptamer-conjugated fluorescence resonance energy transfer nanoparticles for multiplexed cancer cell monitoring. Anal Chem 81: 7009-7014, 2009.

58. Wang L and Tan W: Multicolor FRET silica nanoparticles by single wavelength excitation. Nano Lett 6: 84-88, 2006.

59. Wessels JT, Busse AC, Mahrt J, et al: In vivo imaging in experimental preclinical tumor research - a review. Cytometry A 71: 542-549, 2007

60. Shangguan D, Meng L, Cao ZC, et al: Identification of liver cancer-specific aptamers using whole live cells. Anal Chem 80: 721-728, 2008

61. Davis KA, Abrams B, Lin Y and Jayasena SD: Use of a high affinity DNA ligand in flow cytometry. Nucleic Acids Res 24: 702-706, 1996

62. Bagalkot V, Zhang L, Levy-Nissenbaum E, et al: Quantum dot-aptamer conjugates for synchronous cancer imaging, therapy, and sensing of drug delivery based on bi-fluorescence resonance energy transfer. Nano Lett 7: 3065-3070, 2007.

63. Wang AZ, Bagalkot V, Vasilliou CC, et al: Superparamagnetic iron oxide nanoparticle-aptamer bioconjugates for combined prostate cancer imaging and therapy. ChemMedChem 3: 1311-1315, 2008

64. Kim D, Jeong YY and Jon S: A drug-loaded aptamer-gold nanoparticle bioconjugate for combined CT imaging and therapy of prostate cancer. ACS Nano 4: 3689-3696, 2010.

65. Bander NH: Technology insight: monoclonal antibody imaging of prostate cancer. Nat Clin Pract Urol 3: 216-225, 2006

66. Lupold SE, Hicke BJ, Lin Y and Coffey DS: Identification and characterization of nuclease-stabilized RNA molecules that bind human prostate cancer cells via the prostate-specific membrane antigen. Cancer Res 62: 4029-4033, 2002

67. McNamara JO II, Andrechek ER, Wang Y, et al: Cell type-specific delivery of siRNAs with aptamer-siRNA chimeras. Nat Biotechnol 24: 1005-1015, 2006.

68. Ni X, Zhang Y, Ribas J, et al: Prostate-targeted radiosensitization via aptamer-shRNA chimeras in human tumor xenografts. J Clin Invest 121: 2383-2390, 2011.

69. Farokhzad OC, Jon S, Khademhosseini A, et al: Nanoparticle-aptamer bioconjugates: a new approach for targeting prostate cancer cells. Cancer Res 64: 7668-7672, 2004.

70. Bleickardt E, Argiris A, Rich R, et al: Phase I dose escalation trial of weekly docetaxel plus irinotecan in patients with advanced cancer. Cancer Biol Ther 1: 646-651, 2002.

71. Farokhzad OC, Cheng J, Teply BA, et al: Targeted nanoparticle-aptamer bioconjugates for cancer chemotherapy in vivo. Proc Natl Acad Sci USA 103: 6315-6320, 2006.

72. Cheng J, Teply BA, Sherifi I, et al: Formulation of functionalized PLGA-PEG nanoparticles for in vivo targeted drug delivery. Biomaterials 28: 869-876, 2007.

73. Farokhzad OC, Khademhosseini A, Jon S, et al: Microfluidic system for studying the interaction of nanoparticles and microparticles with cells. Anal Chem 77: 5453-5459, 2005.

74. Gu F, Zhang L, Teply BA, et al: Precise engineering of targeted nanoparticles by using self-assembled biointegrated block copolymers. Proc Natl Acad Sci USA 105: 2586-2591, 2008. 
75. Dhar S, Kolishetti N, Lippard SJ and Farokhzad OC: Targeted delivery of a cisplatin prodrug for safer and more effective prostate cancer therapy in vivo. Proc Natl Acad Sci USA 108 1850-1855, 2011.

76. Ireson CR and Kelland LR: Discovery and development of anticancer aptamers. Mol Cancer Ther 5: 2957-2962, 2006.

77. Soundararajan S, Chen W, Spicer EK, et al: The nucleolin targeting aptamer AS1411 destabilizes Bcl-2 messenger RNA in human breast cancer cells. Cancer Res 68: 2358-2365, 2008.

78. Cao Z, Tong R, Mishra A, et al: Reversible cell-specific drug delivery with aptamer-functionalized liposomes. Angew Chem Int Ed Engl 48: 6494-6498, 2009.

79. Bates PJ, Laber DA, Miller DM, et al: Discovery and development of the G-rich oligonucleotide AS1411 as a novel treatment for cancer. Exp Mol Pathol 86: 151-164, 2009.

80. Mongelard F and Bouvet P: AS-1411, a guanosine-rich oligonucleotide aptamer targeting nucleolin for the potential treatment of cancer, including acute myeloid leukemia. Curr Opin Mol Ther 12: 107-114, 2010.

81. Gendler SJ: MUC1, the renaissance molecule. J Mammary Gland Biol Neoplasia 6: 339-353, 2001.

82. Brockhausen I, Yang JM, Burchell J, et al: Mechanisms underlying aberrant glycosylation of MUC1 mucin in breast cancer cells. Eur J Biochem 233: 607-617, 1995.

83. Reis CA, David L, Seixas M, et al: Expression of fully and under-glycosylated forms of MUC1 mucin in gastric carcinoma. Int J Cancer 79: 402-410, 1998.
84. Ferreira CS, Matthews CS and Missailidis S: DNA aptamers that bind to MUC1 tumour marker: design and characterization of MUC1-binding single-stranded DNA aptamers. Tumour Biol 27: 289-301, 2006.

85. Borbas KE, Ferreira CS, Perkins A, Bruce JI and Missailidis S: Design and synthesis of mono- and multimeric targeted radiopharmaceuticals based on novel cyclen ligands coupled to anti-MUC1 aptamers for the diagnostic imaging and targeted radiotherapy of cancer. Bioconjug Chem 18: 1205-1212, 2007.

86. Ferreira CS, Cheung MC, Missailidis S, et al: Phototoxic aptamers selectively enter and kill epithelial cancer cells. Nucleic Acids Res 37: 866-876, 2009.

87. Savla R, Taratula O, Garbuzenko O and Minko T: Tumor targeted quantum dot-mucin 1 aptamer-doxorubicin conjugate for imaging and treatment of cancer. J Control Release 153: 16-22, 2011.

88. Nolte A, Klussmann S, Bald R, et al: Mirror-design of L-oligonucleotide ligands binding to L-arginine. Nat Biotechnol 14: 1116-1119, 1996.

89. Sayyed SG, Hagele H, Kulkarni OP, et al: Podocytes produce homeostatic chemokine stromal cell-derived factor-1/CXCL12, which contributes to glomerulosclerosis, podocyte loss and albuminuria in a mouse model of type 2 diabetes. Diabetologia 52 : 2445-2454, 2009. 